\title{
Publisher Correction: Proton mediated spin state transition of cobalt heme analogs
}

\author{
Jianping Zhao ${ }^{1,8}$, Qian Peng (10 2,8, Zijian Wang ${ }^{2}$, Wei Xu (1) ${ }^{3}$, Hongyan Xiao (1) ${ }^{4}$, Qi Wu ${ }^{1}$, Hao-Ling Sun ${ }^{5}$, \\ Fang $\mathrm{Ma}^{5}$, Jiyong Zhao ${ }^{6}$, Cheng-Jun Sun ${ }^{6}$, Jianzhang Zhao ${ }^{7} \&$ Jianfeng Li (i) ${ }^{1}$
}

Correction to: Nature Communications https://doi.org/10.1038/s41467-019-10357-z, published online 24 May 2019.

In the original version of this Article, the four structural depictions of orbital distributions were inadvertently omitted from Fig. 5. This has now been corrected in both the HTML and PDF versions of the Article.

Published online: 21 June 2019

\begin{abstract}
cc) reproduction in any medium or format, as long as you give appropriate credit to the original author(s) and the source, provide a link to the Creative Commons license, and indicate if changes were made. The images or other third party material in this article are included in the article's Creative Commons license, unless indicated otherwise in a credit line to the material. If material is not included in the article's Creative Commons license and your intended use is not permitted by statutory regulation or exceeds the permitted use, you will need to obtain permission directly from the copyright holder. To view a copy of this license, visit http://creativecommons.org/licenses/by/4.0/.
\end{abstract}

(C) The Author(s) 2019

\footnotetext{
${ }^{1}$ College of Materials Science and Opto-electronic Technology, CAS Center for Excellence in Topological Quantum Computation, \& Center of Materials Science and Optoelectronics Engineering, University of Chinese Academy of Sciences, Yanqi Lake, Huairou District, 101408 Beijing, China. ${ }^{2}$ State Key Laboratory and Institute of Elemento-Organic Chemistry, College of Chemistry, Nankai University, 300071 Tianjin, China. ${ }^{3}$ Institute of High Energy Physics \& University of Chinese Academy of Sciences, Chinese Academy of Sciences, 100049 Beijing, China. ${ }^{4}$ Key Laboratory of Photochemical Conversion and Optoelectronic Materials, Technical Institute of Physics and Chemistry, Chinese Academy of Sciences, 100190 Beijing, China. ${ }^{5}$ Department of Chemistry and Beijing Key Laboratory of Energy Conversion and Storage Materials, Beijing Normal University, 100875 Beijing, China. ${ }^{6}$ Advanced Photon Source, Argonne National Laboratory, Argonne, IL 60439, USA. 7 State Key Laboratory of Fine Chemicals, School of Chemical Engineering, Dalian University of Technology, West Campus, 2 Ling-Gong Road, 116024 Dalian, China. ${ }^{8}$ These authors contributed equally: Jianping Zhao, Qian Peng. Dedicated to the 40 th anniversary of the University of Chinese Academy of Sciences and the 100th anniversary of Nankai University. Correspondence and requests for materials should be addressed to J.L. (email: jfli@ucas.ac.cn)
} 